\section{Retinal nerve fibre layer imaging compared with histological measurements in a human eye}

EZ Blumenthal', RS Parikh², J Pe'er', M Naik², E Kaliner', MJ Cohen', S Prabakaran², M Kogan' and $\mathrm{R}$ Thomas $^{2}$
Eye (2009) 23, 171-175; doi:10.1038/sj.eye.6702942; published online 24 August 2007

Keywords: retinal nerve fibre layer; histology; morphometry; glaucoma imaging; scanning laser polarimetry; optical coherence tomography

\section{Introduction}

Retinal nerve fibre layer (RNFL) thickness is a key structural measure for diagnosing and following glaucomatous optic neuropathy. While extensively analysed by way of imaging, little is known about histological RNFL thickness patterns. ${ }^{1}$ Only a handful of clinical studies have measured RNFL thickness in post-mortem human ${ }^{2,3}$ and primate eyes. ${ }^{4-7}$ Each of these studies used a different approach to processing the tissue and sectioning the globes, and they differ markedly in the location and grid pattern of the measurements obtained. ${ }^{1}$ In these studies, relatively few RNFL thickness measurements were obtained from each eye, limiting the ability to reconstruct the overall pattern of RNFL thickness at the peripapillary region.

While imaging techniques, as scanning laser polarimetry (SLP) and optical coherence tomography $(\mathrm{OCT})^{8}$ are extensively utilised in clinical practice, their structural validation has been limited. For example, SLP was initially validated and consequently calibrated (arriving at a conversion factor translating degrees of retardation to RNFL thickness in microns) based on two monkey eyes. ${ }^{9}$

Recently, a novel approach to sectioning of the eye was described, ${ }^{10}$ enabling the reconstruction of high-resolution maps of the
${ }^{1}$ Department of Ophthalmology, Hadassah-Hebrew University Medical Center, Jerusalem, Israel

${ }^{2}$ LV Prasad Eye Institute, Hyderabad, India

Correspondence: EZ Blumenthal, Department of Ophthalmology, Hadassah University Hospital, PO Box 12000, Jerusalem 91120, Israel Tel: + 97226776580 ; Fax: +972 26778852 E-mail: eblumenthal@ md.huji.ac.il

Received: 11 September 2006

Accepted in revised form: 7 July 2007

Published online: 24 August 2007 
peripapillary RNFL thickness. This 'umbrella' technique $^{10}$ allows high sampling density of RNFL thickness measurements along each ring section. The histological data obtained with this technique are to a large extent identical in layout, and thus can be compared to thickness data derived using RNFL imaging devices.

It is important to emphasize that a direct (micron for micron) RNFL thickness comparison between histology and imaging is probably unachievable, ${ }^{1}$ owing to both histological and imaging artefacts. Still, qualitative knowledge can be gained from a comparison of these two fundamentally different approaches to RNFL thickness estimation, such that information collected using different approaches can serve to strengthen each other, or question each other, depending on how clustered the results are. To qualify patients for such a comparative study would include advanced neoplastic conditions necessitating exenteration of the orbit, provided that the globe is still conserved, and where no media opacity or retinal abnormalities are present.

\section{Methods}

From a large oculoplastic clinic at LV Prasad, we identified a 51-year old man suffering from spindle-type squamous cell carcinoma infiltrating the orbit, who was scheduled for an orbital exenteration. Standard automated perimetry (white on white) using the SITA standard algorithm, as well as short wavelength (blue on yellow), full threshold 24-2 automated visual fields (VFs) were both within the normal limits.

Human Subject Committee approval and the patient's written consent were obtained, to be imaged before the exenteration and have the globe subjected later to appropriate sectioning and processing. Before surgery, the patient underwent a full eye examination, including slit-lamp biomicroscopy, applanation tonometry, gonioscopy, a dilated fundus examination, fundus photography, and VF testing. None of these tests suggested the presence of glaucoma or other intraocular pathologies. Visual acuity in this eye was 20/25.

Next, the eye was scanned by the following imaging modalities:

(1) SLP with the GDx-VCC (Carl Zeiss-Meditec, Dublin, CA, formerly Laser Diagnostic Technologies, San Diego, CA, USA) with the standard variable corneal compensation (VCC) scanning algorithm, in an undilated pupil.

(2) OCT with the Stratus OCT (Carl Zeiss-Meditec, Dublin, CA, USA), scanning the peripapillary region using the fast RNFL thickness (3.4) algorithm with a pharmacologically dilated pupil.
Quality assurance for these imaging studies included the machine determined quality score, appearance of the scanned image, and searching for various artefacts (such as blur, motion, uneven illumination and/or blinking artefacts). Both these imaging tests were within normal limits in the study eye, which was scheduled to undergo orbital exenteration, as well as in the fellow eye. Unilateral orbital exenteration surgery was later performed as clinically indicated, and the globe was shelled out of the specimen and placed in 5\% phosphatebuffered formalin within 2 min of tissue removal. The fixated globe was sectioned and processed as previously described. ${ }^{10}$

The closed funnel was serially sectioned into $4-\mu \mathrm{m}$ thickness ring sections. Ring diameters were meticulously measured and those ring sections having a diameter of 3.0, 3.5, 4.0, and $4.5 \mathrm{~mm}$ were identified. Each of these four ring sections was photographed and 100 equidistant RNFL thickness measurements were made according to the technique previously described..$^{10}$ All members of the team involved in the histological processing, photographing, measuring, and graphing the data were fully masked to all clinical information, including imaging data.

\section{Results}

A red-free fundus photograph is shown in Figure 1, demonstrating the optic disc appearance and RNFL reflectance pattern, most noticeable along the supero-temporal and infero-temporal arcades. Histologically derived RNFL thickness measurements from four concentric diameter rings, each centred on the optic disc, are presented in Figure $2 \mathrm{a}-\mathrm{d}$. Each plot is

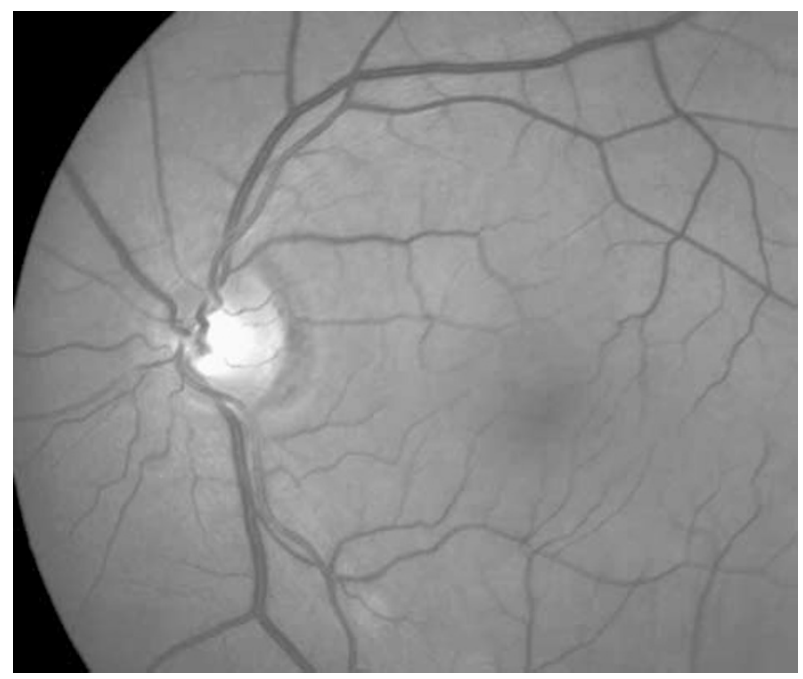

Figure 1 A red-free fundus photograph of the eye. 
a

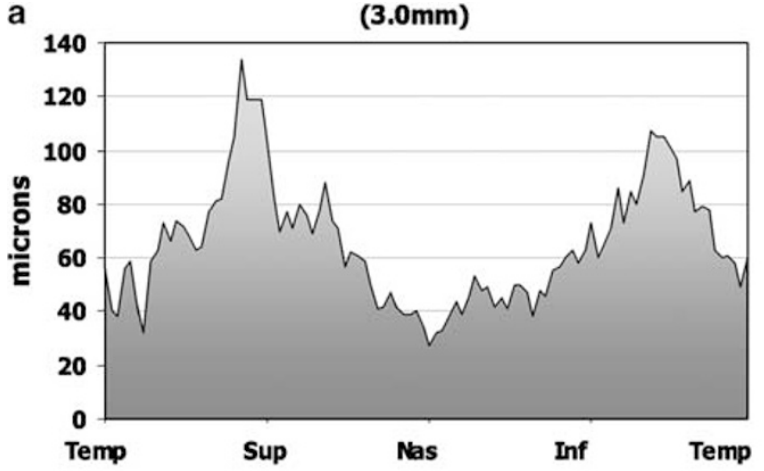

C

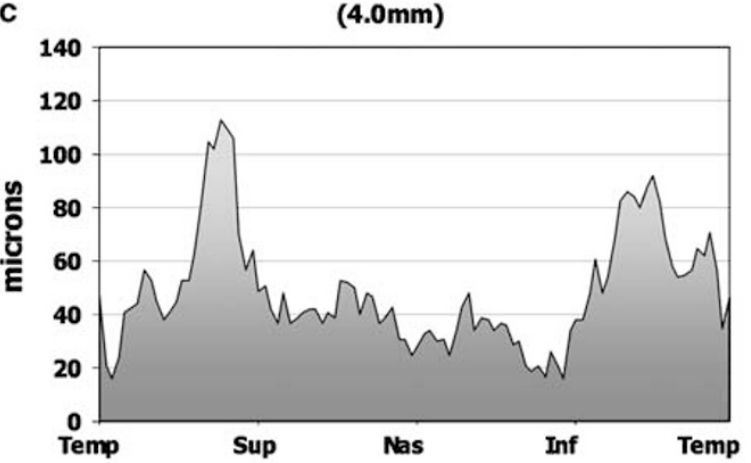

e

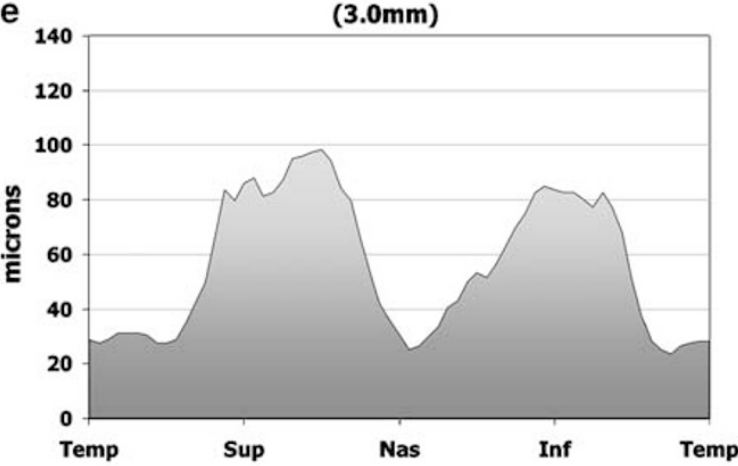

g

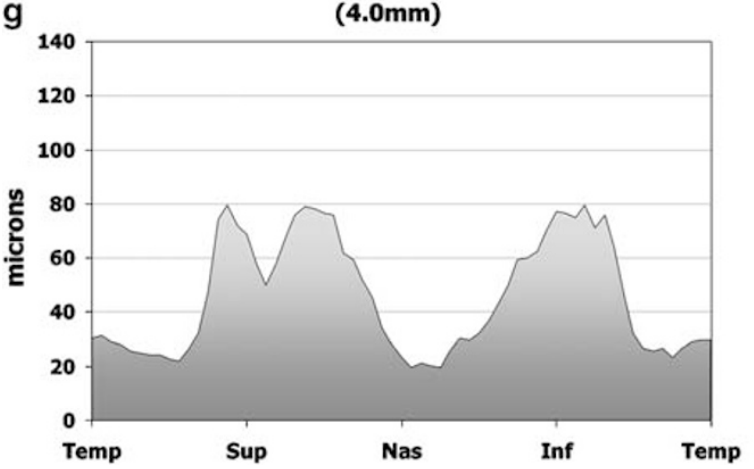

b

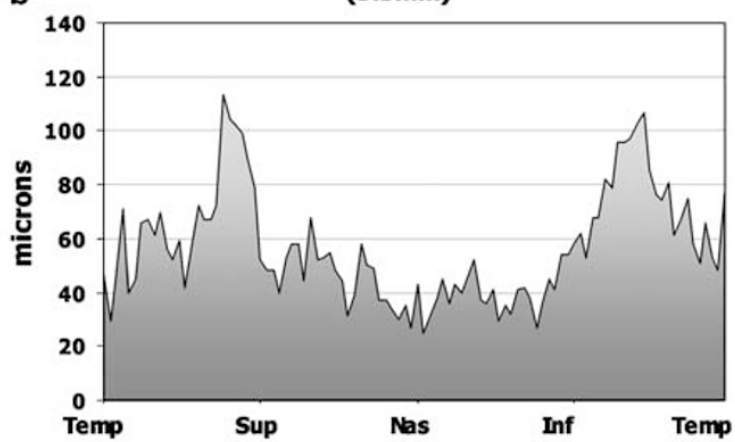

d

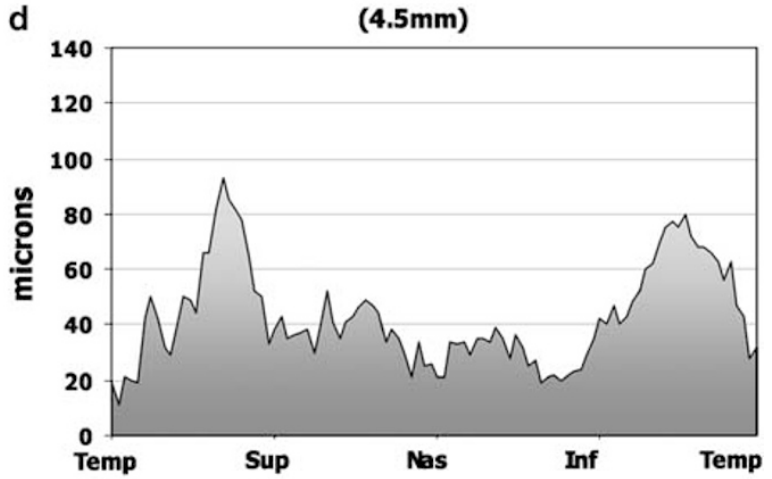

f

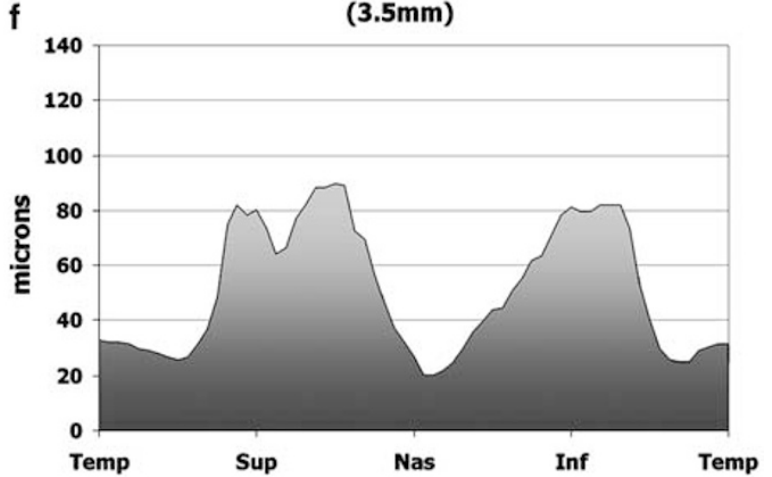

h

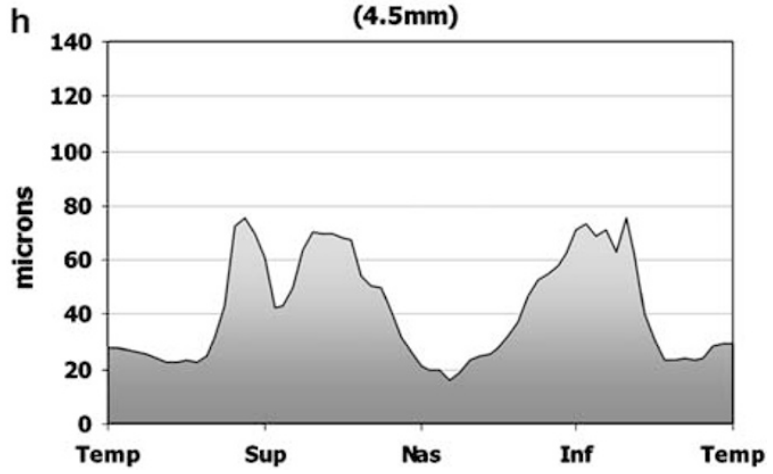

Figure 2 (a-d) RNFL thickness histological measurements. Histologically derived RNFL thickness measurements $\mu \mathrm{m}$ ) along 'ring sections', each centred on the optic disc, following the TSNIT (temporal-superior-nasal-inferior-temporal) pattern, and with diameters of (a) $3 \mathrm{~mm}$, (b) $3.5 \mathrm{~mm}$, (c) $4 \mathrm{~mm}$, (d) $4.5 \mathrm{~mm}$. Each graph is composed of 100 data points. (e-h) GDx-VCC post-processing RNFL thickness. A post-processing of the raw GDx-VCC data shown in Figure 4. The measurement ring was set to different diameter rings matching the diameters chosen in the histological analysis- (a) $3.0 \mathrm{~mm}$, (b) $3.5 \mathrm{~mm}$, (c) $4.0 \mathrm{~mm}$, (d) $4.5 \mathrm{~mm}$. Each graph is composed of 64 data points obtained from the standard exported data file. 
composed of 100 equidistant data points collected along the circumference of one 'ring section'. Figure $2 \mathrm{a}-\mathrm{d}$ demonstrates that the further out the RNFL is from the disc margin, the thinner it is, at every clock hour. Figure 3 presents an OCT fast RNFL thickness algorithm peripapillary scan along a standard measurement ring with a diameter of $3.4 \mathrm{~mm}$. Figure 4 presents a GDx scan with a measurement ring placed at a diameter of 2.8-3.2 mm. Figure 2e-h presents GDx measurements extracted from the scan that served to generate the

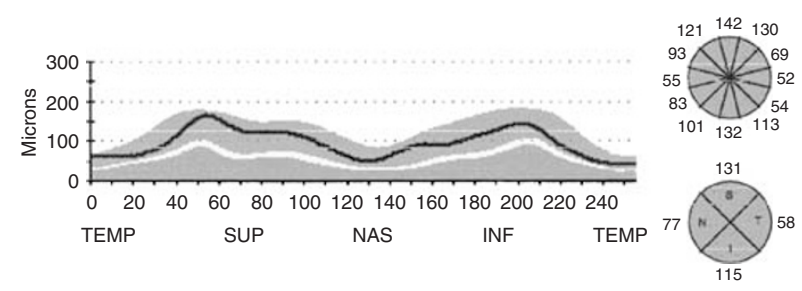

Figure 3 OCT imaging. A stratus OCT scan showing peripapillary RNFL thickness measurements taken along a $3.4 \mathrm{~mm}$ diameter ring centred on the optic disc. printout of Figure 4. However, in Figure $2 \mathrm{e}-\mathrm{h}$, rings identical in diameter to the histological ring sections shown in Figure 2a-d (3.0, 3.5, 4.0, and $4.5 \mathrm{~mm}$ in diameter) are presented. This post-processing was only possible for the GDx since a single GDx scan captures RNFL thickness measurements from an area covering over $20 \times 20$ degrees centred on the optic disc. Owing to this raw data matrix it is possible, on post-processing, to redefine measurement rings of varying sizes.

Histologically derived RNFL thickness $(\mu \mathrm{m})$ at the 3.0 and $3.5 \mathrm{~mm}$ diameter rings (respectively) ranged from a minimum that did not drop below 30, 25, to a maximum of 135, 115 (superiorly) and 110, 105 (inferiorly). In comparison, the $3.0 \mathrm{~mm}$ diameter GDx data, and the $3.4 \mathrm{~mm}$ diameter OCT data (respectively) showed minimum thickness values that did not drop below 25, 40 , while the maximum thickness values extend up to 100, 175 (superiorly) and 85, 140 (inferiorly). The approach taken in this study enables a comparison between histological and GDx data at identical fundus locations.

\begin{tabular}{|c|c|c|}
\hline $\begin{array}{c}\text { TSNIT } \\
\text { Parameters }\end{array}$ & $\begin{array}{c}\text { OD } \\
\text { Actual Val. }\end{array}$ & $\begin{array}{c}\text { OS } \\
\text { Actual Val. }\end{array}$ \\
\hline TSNIT Average & & 57.2 \\
\hline Superior Average & & 68.9 \\
\hline Inferior Average & & 64.9 \\
\hline TSNIT Std. Dev. & & 25.3 \\
\hline \multicolumn{3}{|l|}{ Inter-Eye Symmetry } \\
\hline $\mathrm{NFI}$ & & 16 \\
\hline$p>=5 \%$ & $p<19$ & \\
\hline
\end{tabular}
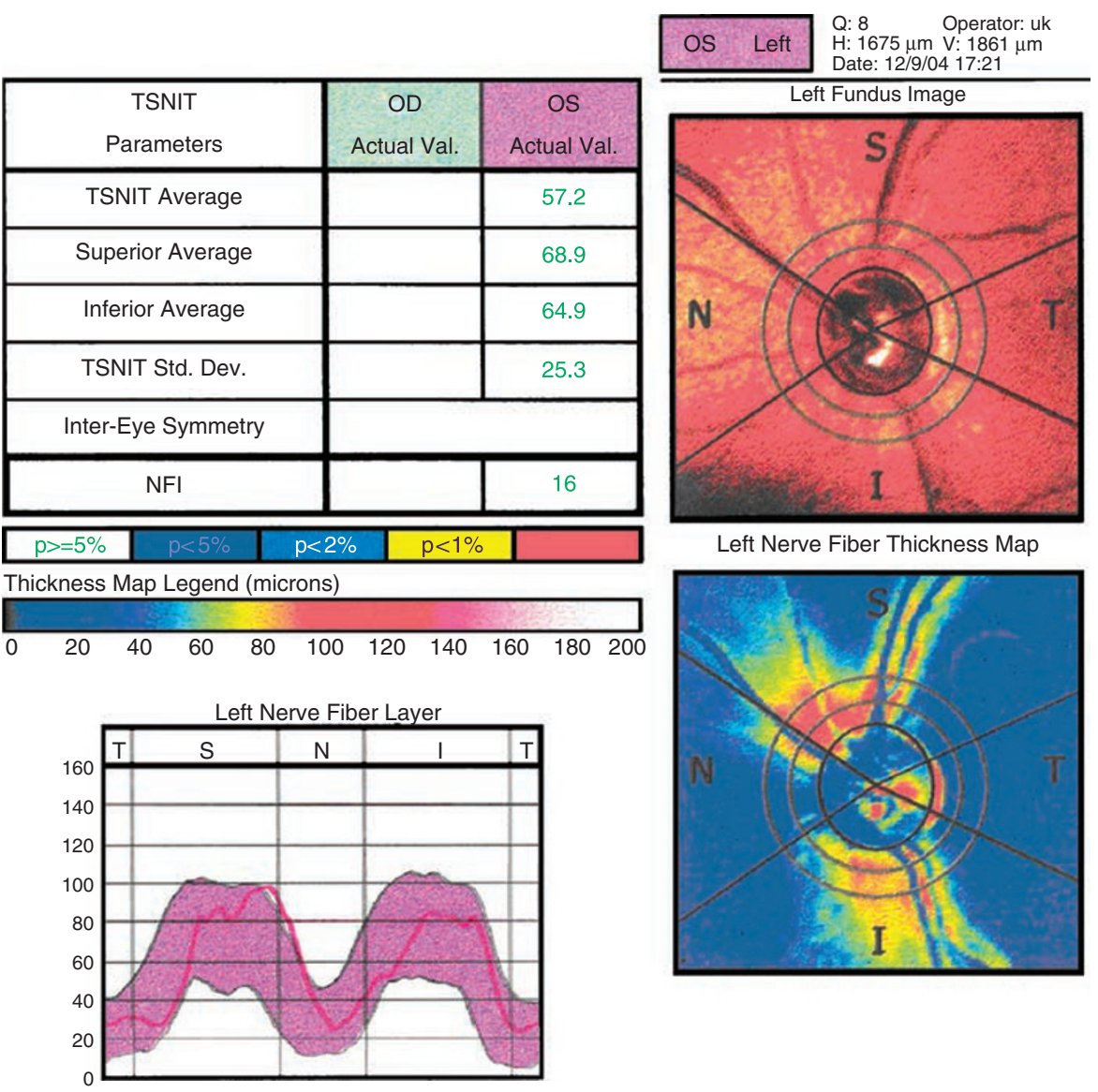

Figure 4 GDx-VCC imaging. A GDx-VCC scan with peripapillary RNFL thickness measurements taken along a 2.8-3.2 mm diameter ring (inner and outer diameters) centred on the optic disc. 


\section{Discussion}

In this novel study, we compare histological RNFL thickness data obtained via the 'umbrella' technique ${ }^{10}$ with imaging data obtained on the same human eye.

RNFL thickness was found to be inversely related to the distance from the centre of the optic disc; we found that at any given clock-hour, the greater the distance from the optic disc border, the thinner the RNFL thickness. RNFL thickness measurements, obtained histologically, confirmed the presence of a 'double hump' pattern with two peaks at the superior and inferior poles. The RNFL thickness (TSNIT) pattern around the disc obtained histologically (Figure $2 \mathrm{a}-\mathrm{d}$ ) resembles the pattern of both OCT (Figure 3) and SLP scans (Figures $2 \mathrm{e}-\mathrm{h}$ and 4 ), although actual micron thickness values differ. Of note, the fundus photo (Figure 1) demonstrates a thicker, superior rim than an inferior one. Likewise, the histological analysis (Figure 2a-d) shows a comparable pattern, with a more robust, superior pole than an inferior one.

Histological-imaging comparisons are important for validating imaging data, especially when substantial discrepancies are found when comparing different imaging modalities. For example, in one study, ${ }^{11}$ an almost two-fold difference in the average RNFL thickness was found between GDx-VCC $(55.26 \pm 4.32 \mu \mathrm{m})$ and Stratus OCT $(101.38 \pm 7.73 \mu \mathrm{m})$ in a group of normal eyes. Of interest, our data on a single eye is completely in line with this GDx-OCT discrepancy, while the histology thickness values were found to be located roughly midway between the GDx and OCT values. Figure $2 \mathrm{a}-\mathrm{h}$ enables a side-by-side comparison between histology and GDx-VCC data, for equal diameter rings. For example, a comparison of Figure $2 \mathrm{a}$ and e (both at the $3.0 \mathrm{~mm}$ diameter) shows a rather similar modulation pattern for histology as compared to GDx-VCC.

This study demonstrates that a histological-imaging comparison can be achieved on human eyes. Many more eyes would be needed to conduct a quantitative analysis aimed towards validation of imaging devices. More so, such a comparison would be partially limited by artefacts inherent to any histological technique chosen ${ }^{1}$ as well as to imaging artifacts and limitations. ${ }^{11}$ Histological artefacts may include shrinkage, expansion, and distortion of tissue secondary to post-mortem changes, processing, and staining of the tissue. Imaging artefacts may include errors in scan location, non-compensated magnification, and most important, erroneous conversion factors, which are themselves based on limited histological specimens with their own inherent artefacts. A potential limitation of this study relates to the fact that the enucleated study eye might have been affected by the orbital tumour, and hence it is doubtful whether this was indeed a perfectly healthy eye. Of note, extensive structural and functional diagnostic tests were performed on this eye before enucleation, and none have suggested the possibility of glaucomatous optic neuropathy or RNFL damage. Additionally, since this is a study comparing histology to imaging at a given point in time, histology-imaging correlation can be evaluated regardless of the diagnostic status of the eye.

\section{Acknowledgements}

This study was supported by a grant from Laser Diagnostic Technologies Incorporated.

\section{References}

1 Frenkel S, Morgan JE, Blumenthal EZ. Histological measurement of retinal nerve fiber layer thickness. Eye 2005; 19: 491-498.

2 Varma R, Skaf M, Barron E. Retinal nerve fiber layer thickness in normal human eyes. Ophthalmology 1996; 103: 2114-2119.

3 Dichtl A, Jonas JB, Naumann GO. Retinal nerve fiber layer thickness in human eyes. Graefes Arch Clin Exp Ophthalmol 1999; 237: 474-479.

4 Radius RL. Thickness of the retinal nerve fiber layer in primate eyes. Arch Ophthalmol 1980; 98: 1625-1629.

5 Quigley HA, Addicks EM. Quantitative studies of retinal nerve fiber layer defects. Arch Ophthalmol 1982; 100: 807-814.

6 Ogden TE. Nerve fiber layer of the primate retina: thickness and glial content. Vision Res 1983; 23: 581-587.

7 Morgan JE, Waldock A, Jeffery G, Cowey A. Retinal nerve fibre layer polarimetry: histological and clinical comparison. Br J Ophthalmol 1998; 82: 684-690.

8 Huang D, Swanson EA, Lin CP, Schuman JS, Stinson WG, Chang W et al. Optical coherence tomography. Science 1991; 254: 1178-1181.

9 Weinreb RN, Dreher AW, Coleman A, Quigley H, Shaw B, Reiter K. Histopathologic validation of Fourier-ellipsometry measurements of retinal nerve fiber layer thickness. Arch Ophthalmol 1990; 108: 557-560.

10 Blumenthal EZ. Quantifying retinal nerve fiber layer thickness histologically: a novel approach to sectioning of the retina. Invest Ophthalmol Vis Sci 2004; 45: 1404-1409.

11 Leung CK, Chan WM, Chong KK, Yung WH, Tang KT, Woo J et al. Comparative study of retinal nerve fiber layer measurement by stratus OCT and GDx VCC, I: correlation analysis in glaucoma. Invest Ophthalmol Vis Sci 2005; 46: 3214-3220. 